\title{
An Evaluation of Citizen Science Smartphone Apps for Inland Water Quality Assessment
}

\author{
Tim J. Malthus ${ }^{1, *(\mathbb{D}}$, Renee Ohmsen ${ }^{2}$ and Hendrik J. van der Woerd ${ }^{3(\mathbb{C}}$ \\ 1 Coastal Sensing and Modelling Group, Coasts Program, CSIRO Oceans and Atmosphere, \\ Ecosciences Precinct, 41 Boggo Road, Dutton Park, QLD 4102, Australia \\ 2 School of Earth and Environmental Sciences, University of Queensland, St Lucia, QLD 4072, Australia; \\ renee.ohmsen@uqconnect.edu.au \\ 3 Institute for Environmental Studies (IVM), VU University Amsterdam, De Boelelaan 1111, \\ 1081 HV Amsterdam, The Netherlands; hans.van.der.woerd@ivm.vu.nl \\ * Correspondence: tim.malthus@csiro.au
}

Received: 28 March 2020; Accepted: 13 May 2020; Published: 15 May 2020

check for updates

\begin{abstract}
Rapid and widespread monitoring of inland and coastal water quality occurs through the use of remote sensing and near-surface water quality sensors. A new addition is the development of smartphone applications (Apps) to measure and record surface reflectance, water color and water quality parameters. In this paper, we present a field study of the HydroColor (HC, measures RGB reflectance and suspended particulate matter (SPM)) and EyeOnWater (EoW, determines the Forel-Ule scale-an indication to the visual appearance of the water surface) smartphone Apps to evaluate water quality for inland waters in Eastern Australia. The Brisbane river, multiple lakes and reservoirs and lagoons in Queensland and New South Wales were visited; hyperspectral reflection spectra were collected and water samples were analysed in the laboratory as reference. Based on detailed measurements at 32 sites, covering inland waters with a large range in sediment and algal concentrations, we find that both water quality Apps are close, but not quite on par with scientific spectrometers. EoW is a robust application that manages to capture the color of water with accuracy and precision. $\mathrm{HC}$ has great potential, but is influenced by errors in the observational procedure and errors in the processing of images in the iPhone. The results show that repeated observations help to reduce the effects of outliers, while implementation of camera response functions and processing should help to reduce systematic errors. For both Apps, no universal conversion to water quality composition is established, and we conclude that: (1) replicated measurements are useful; (2) color is a reliable monitoring parameter in its own right but it should not be used for other water quality variables, and; (3) tailored algorithms to convert reflectance and color to composition could be developed for lakes individually.
\end{abstract}

Keywords: citizen science; smartphone; water quality; lakes; EyeOnWater; HydroColor; Australia

\section{Introduction}

Surface freshwater is a finite resource essential for human and ecosystem existence. Adequate freshwater quality and quantity is required for sustainable development necessary for human consumption, irrigation, fishing and recreational use [1,2]. Access to water with good quality is one of the 17 sustainable development goals [3]. Water quality is an important aspect of Australia's freshwater resources and ongoing information about Australia's water quality is vital for water resource management. Studies [4-6] have evaluated the contribution and application of remote sensing for providing freshwater monitoring of biophysical properties within the water column. 
Of all the biological, chemical and physical parameters of natural waters, the detection of the photosynthetic pigment chlorophyll a (CHL) is prominent. CHL is a molecule present within most algal groups, including cyanobacteria and is commonly used as a universal index for phytoplankton biomass [7]. If present in sufficient quantity, CHL has a very prominent fingerprint on the absorption and reflectance properties of water and can be detected by sensors that operate in the visual domain $(400-720 \mathrm{~nm})[8,9]$. The two other components that leave an imprint on water reflectance are suspended particulate matter (SPM) and the colored fraction of dissolved organic matter (CDOM). SPM is a combination of algal (detritus) and non-algal (sediment) concentrations [10]. Even in small concentrations, SPM dominates the light availability (turbidity) in the water and the strength of the reflected optical signal above water [11,12]. Similarly, high concentrations of humic and fulvic acid compounds in the water strongly absorb blue light and give these waters a dark impression with a brown to yellow color $[10,13]$.

To monitor the thousands of natural lakes, freshwater reservoirs, billabongs and river systems in Australia, a system is required that is able to access these waters with high spatial and temporal resolution. For example, harmful excess CHL levels in inland waters, also referred to as algal blooms, can develop in days to weeks and blooms can be patchy and localized (Figure 1). In Australia, water quality monitoring is undertaken at the state and territory levels or by agencies such as catchment management authorities and local governments, private utilities and mining companies across privately owned waters and affected public waters. Coverage is patchy in both space and time, and is dependent on individual agency priorities and resources and uses variable methods; most importantly, public data availability is remarkably inconsistent [14]. At least four lakes (Atkinson, Dyer, Liddell and Einbunpin) reported on in this paper have experienced closure for water contact due to high toxic cyanobacteria levels.

Automated or hand-held hyperspectral sensor systems may be used to derive water quality parameters and make a rapid assessment of harmful events, such as the presence of algal blooms [15]. In the last decade, multiple studies have shown that coupling of in situ spectral measurements to satellite data leads to an inland water monitoring system with high potential [16-22]. In Australia, the first steps towards such a system have been taken [23,24].

Wernand et al. [25] introduced a new parameter that can be retrieved accurately from the MERIS ocean color sensor-the Forel-Ule index (FUI). This FUI is a discrete number system developed at the start of the 20th century to quantify the color of water, as perceived by the human eye. In two subsequent articles, Van der Woerd and Wernand $[26,27]$ introduced the hue angle as the quantitative and universal color parameters that can be derived from ocean color sensors and from moderate-resolution sensors such as Landsat-8 and Sentinel-2. In a ground-breaking article, Wang et al. [28] demonstrated that the worldwide monitoring of the eutrophic state of inland waters with the Moderate Resolution Imaging Spectroradiometer (MODIS) instrument is possible, based on the hue and the FUI. Recently, quantification of the color characteristics of inland waters by satellite-derived color has been applied at the national level for New Zealand [29] and Italy [30]. 

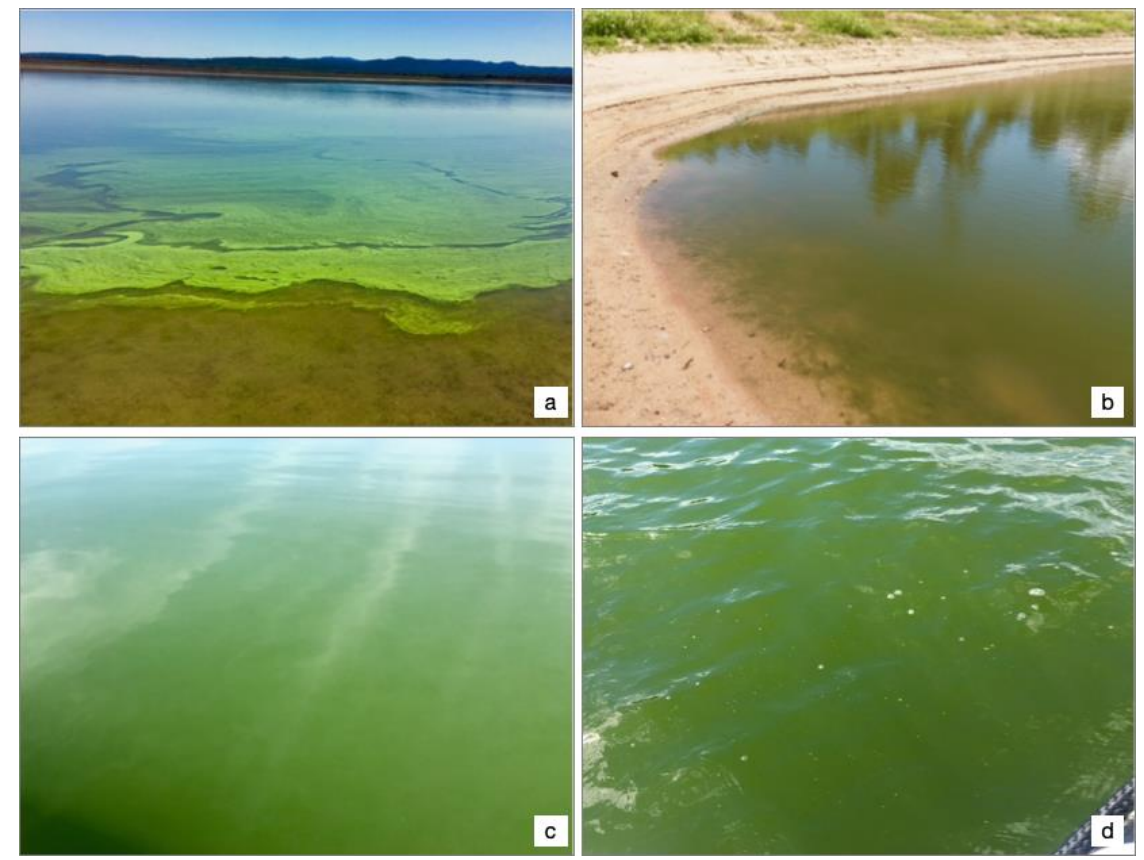

Figure 1. (a) Lake Clarendon, with an example of surface scum caused by high concentrations of cyanobacteria; (b) Lake Dyer and the impact of cyanobacteria on recreational sites, particularly how it impacts on aesthetic values. Varying levels of cyanobacteria in (c) Lake Liddell and (d) Lake Baroon.

In 2014, the EyeOnWater (EoW) and HydroColor (HC) smartphone applications (Apps) became available to measure the color of water. The use of smartphones in the hands of concerned citizens to monitor water quality may provide a real-time estimation of water quality, complementing laboratory sample analysis and the spectral systems discussed above and meeting the need for greater spatial coverage [21]. In both Apps, the camera on the smartphone is used to take Red-Green-Blue (RGB) color images of the water surface. Models are then applied to derive parameters such as hue angle, the FUI, SPM or turbidity.

The EoW App (www.eyeonwater.org) was developed in the European Citclops project. It has been calibrated and tested in Dutch inland waters with a large diversity in composition [31]. The HC App (http://misclab.umeoce.maine.edu/research/HydroColor.php) was tested extensively in both coastal and inland US waters [32] and British Columbia [33]. Besides a limited test in the Kesses Dam, Kenya [34], a systematic comparison of both Apps has not been published.

In this article, we evaluate the accuracy and efficiency of EoW and HC measurements on a larger range of water bodies within an Australian context. A set of optical and laboratory water measurements over a range of water qualities (including bloom conditions) from water bodies in South East Queensland and New South Wales was collected. The accuracy and precision of water color measurements for water quality from the two smartphone applications for citizen science were referenced to reflectance measurements made with a standard underwater spectroradiometer. Finally, and where appropriate, the potential for water color measurements to derive other water quality parameters is discussed.

\section{Materials and Methods}

\subsection{Study Area and Measurement Campaign}

This study was carried out using a total of thirteen inland water bodies within Australia-ten from South East Queensland (SEQ) and three from Northern New South Wales (NSW). These particular water bodies were chosen due to their variation in visible water quality, ease of access and location 
(Figure 2). With the exception of the urban Einbunpin Lagoon and Brisbane River, all other lakes sampled sit within predominantly mixed agricultural and forested catchments. In total, 32 sites were visited between February 2016 and May 2016, where reflectance measurements and water samples were collected between 10:00 and 14:00 h local time. At each of the sites, water samples were taken alongside hyperspectral reflectance measurements made using a Satlantic spectroradiometer, and measurements made using the HC and EoW Apps operated using either an iPhone 6 or iPhone 6s. Three replicates were taken at each station to examine the variation in successive measurements. At some stations two observers operated the EoW and HC Apps simultaneously. The smartphone cameras were also used to record general sky conditions, horizontal visibility, surface wave action and overall variation in water color. Ancillary measurements were recorded at each site following methods in [35]. Measurements included the condition of the sky, wind speed and direction, wave height, Secchi disk depth and bottom depth. The campaign stations are summarized in the first four columns of Table S1. The exact GPS coordinates of all stations, time of recording and their position in the Lakes are recorded on the EoW website (https://www.eyeonwater.org).

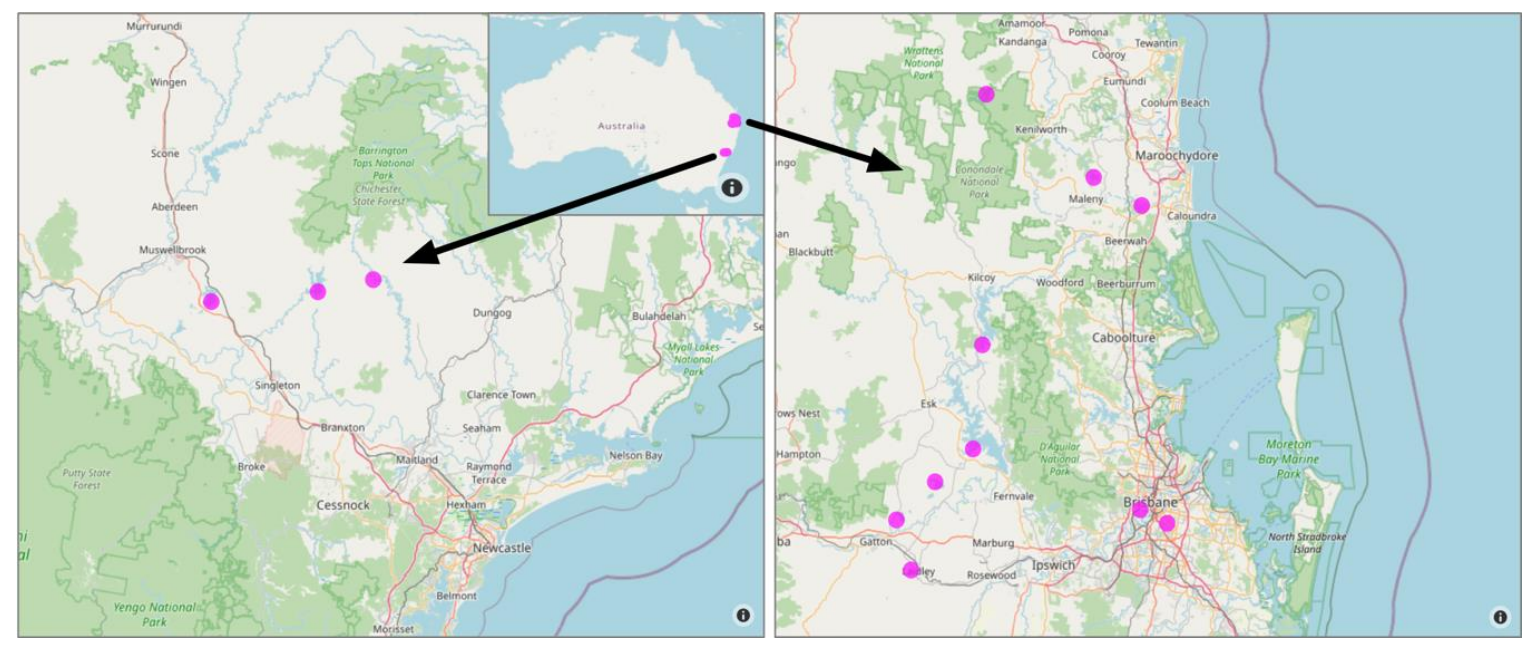

Figure 2. The locations of the 13 water bodies sampled in New South Wales and South East Queensland. Basemap courtesy of openstreetmap.org.

\subsection{Water Sampling}

Water samples were stored in a darkened cooler with ice packs until filtration and processing. Storage time to the laboratory did not exceed $4 \mathrm{~h}$ and all samples were filtered within $24 \mathrm{~h}$ of collection, following the REVAMP protocols [36]. For SPM, Whatman GF/F filters were prepared and weighed prior to our use. Each sample was filtered in triplo. Depending upon the turbidity of the water, between 200 and $500 \mathrm{~mL}$ of sample water was filtered under low vacuum pressure to prevent the particles from breaking up. Filters for SPM were stored in separate dishes for gravimetric analysis following drying. CHL filters folded into a cryo-vial, covered in aluminium foil and stored in a $-80^{\circ} \mathrm{C}$ freezer. For CDOM, samples were filtered using the Millipore filter units and a $0.22 \mu \mathrm{m}$ polycarbonate filter (Millipore). Approximately $80 \mathrm{~mL}$ of sample was filtered through the unit. This water was used to rinse the filter bottle and the unit. Approximately $100 \mathrm{~mL}$ was then filtered; $80 \mathrm{~mL}$ of this filtered water was placed in a small acid-washed glass bottle covered in aluminium foil and placed in the fridge. Water quality samples, once filtered and prepared, were analysed at CSIRO Oceans and Atmosphere's Hobart laboratories for determination of, amongst others, CHL, SPM and CDOM. The methodology for the analysis of water quality samples followed in detail those in [37]. Results are documented in Table S1. 


\subsection{Remote Sensing Reflectance}

A Satlantic HyperOCR hyperspectral radiance radiometer $\left(8.5^{\circ}\right.$ field of view) was used to collect reflectance data in over 136 spectral channels over the 350 to $800 \mathrm{~nm}$ wavelength range at a sampling rate of $3 \mathrm{~Hz}$. The radiance radiometer was pointed downwards consecutively over a 10\% calibrated Spectralon ${ }^{\mathrm{TM}}$ panel, $20 \mathrm{~cm}$ over the water surface ( $\sim 3 \mathrm{~cm}$ footprint) and just under the surface (Figure 3 ). Generally, 70-90 scans were recorded over a $30 \mathrm{~s}$ interval for each measurement position. The three different measurement geometries are required to characterize the spectra of the main components in the light field: the total flux of photons from sun and sky that enter the water $\left(E_{d}\right.$ in $\left.W^{-2} s^{-1}\right)$, sky radiance $\left(L_{s}\right.$ in $W m^{-2} s^{-1} s^{-1}$ ) that is reflected at the water surface (at depth 0 ) and the radiance from the water $\left(\mathrm{L}_{\mathrm{W}}\right.$ in $\left.\mathrm{W} \mathrm{m} \mathrm{m}^{-2} \mathrm{~s}^{-1} \mathrm{sr}^{-1}\right)$.

This measurement method was adopted to address the limitations faced with using a small boat for field data collection [15] and we were fortunate enough to apply this method due to fine weather and stable water surface. The method we used has recently been shown to 'demonstrate ample equivalence' to near-surface above-water nadir-view measurements performed by shielding the sky/sun glint contributions [38], the latter equivalent to the 'sky blocked' approach recommended in [39]. The method draws on a long heritage of similar reflectance measurements made in inland waters [4,40-44] and forms the basis of many measurements in studies which have used aggregated reflectance data across multiple campaigns $[45,46]$. Noise was reduced by taking 60-90 spectra, and removing outliers, thereby removing the effects of changing illumination conditions in the atmosphere and ripples at the air-water interface. We checked the variability about the individual measurements caused by the influence of wave action at a turbid and 'wavy' site and found coefficients of variation of $\sim 4.2 \%$ for the panel measurements and $\sim 2 \%$ for the water surface measurements (Figure S1). These results are well within the acceptable uncertainties for such measurements despite what configuration of sensors and viewing geometries is used [39].

Hue angle is not dependent upon the absolute $\operatorname{Rrs}\left(\mathrm{sr}^{-1}\right)$; it is purely determined by the relative distribution of Rrs over the visible wavelengths. However, to further check the impact of the depth on measurements of the relative distribution of Rrs and hue angle, we used HydroLight ${ }^{\mathrm{TM}}$ modelling to show that the impact on hue angle determination is extremely small (within 0.2 degrees for a sensor immersed $5 \mathrm{~cm}$ below the water surface, Figure S2).
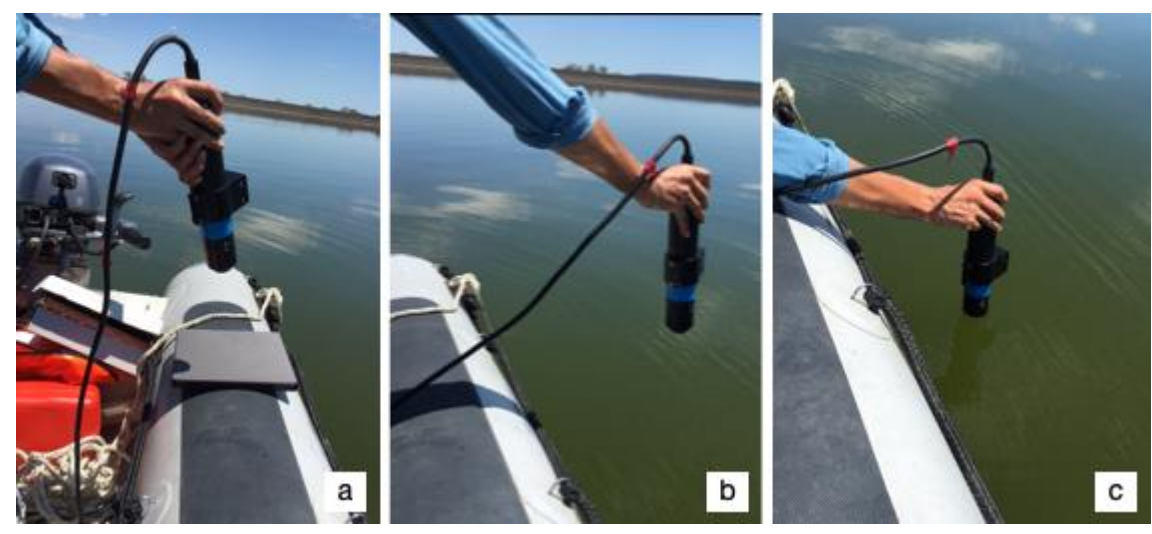

Figure 3. Deployment of the Satlantic spectroradiometer at Lake Dyer. Three sets of successive measurements were taken (a) over the calibrated grey panel, (b) over the water surface and (c) just below the surface.

The above-surface remote-sensing reflectance $\operatorname{Rrs}\left(\mathrm{sr}^{-1}\right)$ is calculated in accordance with the methods of [47]. This property is a function of wavelength $(\lambda)$ and defined by dividing the upward water leaving radiance $\left(\mathrm{L}_{W}\right)$ by the downward irradiance $\left(\mathrm{E}_{\mathrm{d}}\right)$, just above the surface, indicated by $\left(0^{+}\right)$:

$$
\operatorname{Rrs}(\lambda)=\mathrm{L}_{\mathrm{w}}\left(0^{+}, \lambda\right) / \mathrm{E}_{\mathrm{d}}\left(0^{+}, \lambda\right)
$$


The $E_{d}\left(0^{+}, \lambda\right)$ was calculated from the grey Spectralon ${ }^{T M}$ panel radiance $\left(L_{d}\right)$ :

$$
E_{d}\left(0^{+}, \lambda\right)=\beta \pi L_{d}(\lambda)
$$

where $\beta$ is the calibration correction (10) for the $10 \%$ reflectance panel and $\pi$ accounts for the conversion from radiance to irradiance. Because the tip of the Satlantic spectrometer was immersed in the water, we could derive the $L_{w}\left(0^{+}, \lambda\right)$ directly from underwater radiance $L_{w}\left(0^{-}, \lambda\right)$ and correcting for the refraction effects by dividing by $n^{2}$, with $n=1.33$ the refractive index of freshwater. The very small Fresnel reflection at $0^{\circ}$ from water to air was ignored.

To be comparable to the images collected by the HC and EoW Apps, Satlantic data of Rrs $(\lambda)$ were converted to RGB [27] and references therein. The RGB spectral response of the human observer is well characterized and described by the standard colorimetric 2-degree Color-Matching Functions (CMF) of the International Commission on illumination [48]. First, both the Satlantic-derived $\operatorname{Rrs}(\lambda)$ and the CIE RGB curves were interpolated on a $1 \mathrm{~nm}$ grid, multiplied and integrated over all wavelengths. This resulted in the 3 chromaticity values XYZ (describing the RGB values in that order). Then, the red $(x)$ and green $(y)$ normalized value was derived by dividing by $X+Y+Z$. Grey or white light, where there is no wavelength dependency, has coordinates $x=1 / 3$ and $y=1 / 3$-the white point. Finally, the distance to the white point (saturation) and the hue angle were calculated. In this paper, the hue angle is expressed in degrees counter-clockwise relative to the $y=1 / 3, x=1 / 3$ to 1.0 line (hue angle zero). Further information is available in [31,49] and [28], including the transformation table from hue angle to the FUI.

\subsection{The HC Application}

Developed by Leeuw from the University of Maine [50], HC is a water quality App that uses an iPhone's digital camera to determine the Rrs of the water body in the RGB channels. HydroColor uses images collected by the user to measure how much light is reflected from the surface water, corrected for the reflection of sky radiance $\left(L_{s}\right)$ off the surface and normalized to incoming radiation $\left(E_{d}\right)$. In fact, $\mathrm{HC}$ assumes that the camera acts as a linear spectrometer and that the same protocol can be followed as for standard above-water radiometer settings [8,36,51]. The absolute magnitude of the reflectance in these channels are used to estimate SPM concentrations and the turbidity of the body of water using in-built algorithms [32,50]. In this paper, we used the HC App version available at the start of 2016.

HC is well designe and, has clear instructions and easy to read pages. HC uses the internal GPS and clock to compute the position to the sun. With the help of the smartphone's compass and gyroscope, the user is supported in finding the optimum viewing angles to minimize surface reflection [52]. Examples of the HC screens are given in Figure 4. The observer must possess an 18\% photographers' grey card to normalize to the ambient illumination. The App compiles information for latitude, longitude, date, time, sun zenith, sun azimuth, device zenith, device azimuth, exposure values, remote sensing reflectance, turbidity, suspended particulate matter, concentration and the backscattering coefficient [32,50]. HC takes a $200 \times 200$ pixel square in the centre of the image and averages these RGB values. The digital numbers (DNs) in each band are divided by the camera exposure time $\left(\mathrm{DN} \mathrm{s}^{-1}\right)$ as an approximation to the radiance. The Rrs in RGB is then calculated using the relative radiance measured in each image:

$$
\operatorname{Rrs}(R G B)=\left(L_{u}(R G B)-\rho . L_{s}(R G B)\right) / E_{d}(R G B)
$$

where $L_{u}(R G B)$ is water surface radiance $\left(D N ~^{-1}\right), L_{s}(R G B)$ is the sky radiance $\left(D^{2} ~ s^{-1}\right.$ ) and $\rho$ is the sun/sky glint coefficient at the air-water interface, taken as 0.028 from [53]. The $E_{d}$ (RGB) is derived from the grey card observation using Equation (2), where in this case $\beta=1 /(0.18)$. Note that although the input parameters are measured in $\mathrm{DN} \mathrm{s}^{-1}$, the output has units $\left(\mathrm{sr}^{-1}\right)$. Finally, HC uses two non-linear models to convert the Rrs in the R-band to SPM concentration and subsequently to translate 
the SPM concentration to turbidity; Leeuw and Boss (2018) outline the underlying assumptions and restrictions in these models.
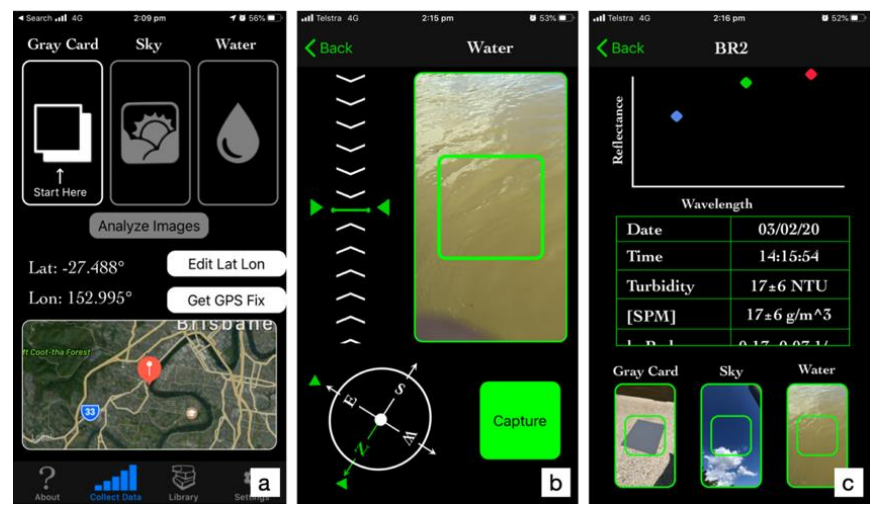

Figure 4. The three steps involved in capturing data on the Brisbane river with the HydroColor (HC) application. (a) The opening screen as 'start here,' suggesting the starting photo. The first photo is of a grey card, the second photo taken is of sky and the third photo is of the water (shown in $\mathbf{b}$ ). The results are summarized in (c).

Two values are presented by the application (Figure $5 b$ ). One is the FUI selected by the user (FU_Value), achieved by matching the photo taken with the appropriate color hue. The second value (FU_Processed) is based on automated processing of the uploaded image by the WAter COlor from Digital Images (WACODI) algorithm on the EoW server [31]. WACODI assumes that all images from commercial smartphones are delivered in the sRGB standard format. Conversion to linearized chromaticity coordinates $\mathrm{XYZ}$ is performed in two steps: (1) convert the sRGB values in the digital images to the XYZ CIE system using the conversion matrices found in [54] and (2) Gamma correction to compensate for non-linear compression of images in the sRGB system. In the image, 16 small sections of $40 \times 40$ pixels are selected and the resulting distribution of hue angles in all sections are combined to select the 'best' approximation of the water hue and the corresponding FUI [31]. As images can be quite complex, with shadows, the rim of a boat or pier, the 'best' selection is not always the 'centre' section of the image. In this paper, we used the WACODI open-source software [31] to analyse all images in more depth. In total, $6 \times 6$ subsections were analysed and four hue angles were retrieved: the 'best' and 'centre' hue angle, assuming sunny or overcast illumination conditions.

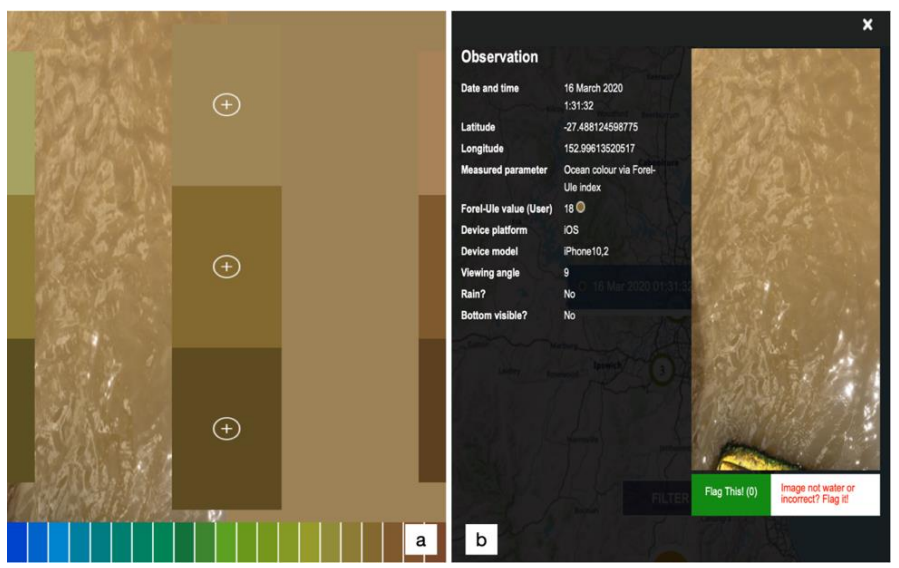

Figure 5. At the proper orientation, a photo is first taken and a color chart appears. The user is able to scroll through the color chart and select which color best matches the photo of the water (a). After uploading and processing on the EyeOnWater (EoW) server, the photo with all results is available on the webserver (b). 


\section{Results}

\subsection{Water Quality Conditions}

In Table S1, a summary of the mean laboratory results for CHL and SPM concentrations is given, together with the CDOM absorption at $440 \mathrm{~nm}$. The results show that the measurements taken across the 13 water bodies have covered a wide range of water qualities from oligotrophic (poor nutrient supply and little plant life) to hypertrophic (high nutrient content and water quality issues). The spread of CHL levels suggests that the majority of sites included within this study have relatively low chlorophyll a concentrations. The majority of sites are within the $1.5 \mu \mathrm{g} / \mathrm{L}$ (Lake St Clair) to $26 \mu \mathrm{g} / \mathrm{L}$ (Lake Liddell) CHL range with sites such as Lake Dyer, Einbunpin and Atkinson Dam presenting elevated levels of 177, 121 and $85 \mu \mathrm{g} / \mathrm{L}$, respectively. Secchi Disk depth ranges from the clearer blue waters of Lake St Clair $(5 \mathrm{~m})$ to the murky yellow-green water of Lake Atkinson $(0.27 \mathrm{~m})$. The SPM results further reflect this where SPM ranges from the clearer waters of Lake St Clair $(0.89 \mathrm{mg} / \mathrm{L})$ to the highly turbid Brisbane River $(158 \mathrm{mg} / \mathrm{L})$. CDOM ranged from $0.34 \mathrm{~m}^{-1}$ at Lake Liddell to $2.30 \mathrm{~m}^{-1}$ at Einbunpin Lagoon.

\subsection{Remote Sensing Reflectance}

Most of the waters were calm, with no or only small ripples; the weather was calm and sky conditions ranged from cloudless to almost overcast (see Table S1). In Figure 6, the Rrs ( $\lambda$ ) of 31 sites across 13 water bodies are shown, normalized to the Rrs $(550 \mathrm{~nm})$. The reflectance curve is impacted by particles and dissolved matter (CHL, SPM and CDOM) within the water column, in particular suspended algal biomass (CHL), suspended sediment and CDOM. The spectra from the water bodies measured show several common features. With the exception of the Brisbane River, all sites have a peak between 560 and $580 \mathrm{~nm}$, corresponding to scattering due to the presence of particulate matter and low absorption. There is a prominent reflectance peak at approximately $700 \mathrm{~nm}$ for the sites with the highest chlorophyll levels—-these sites include Einbunpin Lagoon, Lake Atkinson and Lake Dyer. This high peak is caused by an interaction of algal absorption in the red at $676 \mathrm{~nm}$, in combination with scattering by algae and pigment [55]. The absorption near $620 \mathrm{~nm}$ is a result of the phycocyanin (PC) pigment and suggests the presence of blue-green algae. Sites with steeper slopes in the green region of the spectrum, such as Lake Liddell have significantly higher PC levels compared to Lake St Clair which has a gradual slope indicating a lower PC value. Since the red band (X) has a FWHM between 558 and $634 \mathrm{~nm}$, it is clear from these spectra that waters with high CHL concentrations corrupt any simple relation between X (or Y) and SPM [56]. The results of the conversion of these complex spectra to hue angle are shown in Table S1.

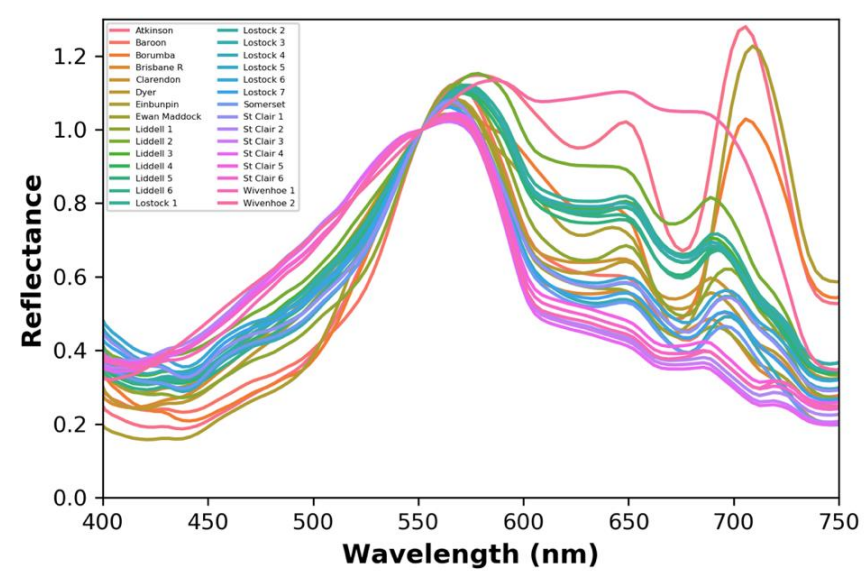

Figure 6. Satlantic Rrs spectra from the 13 water bodies measured, normalized to $550 \mathrm{~nm}$. 


\subsection{EoW Performance}

During the campaign, a total of 110 measurements of the 13 waters were uploaded to the EoW website, with a minimum of three replicates taken at each of the locations. These can be found on the EoW website, with the location and timing provided in Table S1. Each entry shows the FU_Value assigned by the user and FU_Processed from the image (see Figure 5b). In Figure 7, three examples of these are shown, which demonstrate the variability in water composition, illumination and interfering features in the image, such as shadow, sun glint and boat rim. Some images were taken from the shore at $1.70 \mathrm{~m}$ height and sometimes the use of a small boat only allowed images to be taken $30 \mathrm{~cm}$ above the water surface. All images were analysed and no visual inspection or selection took place.
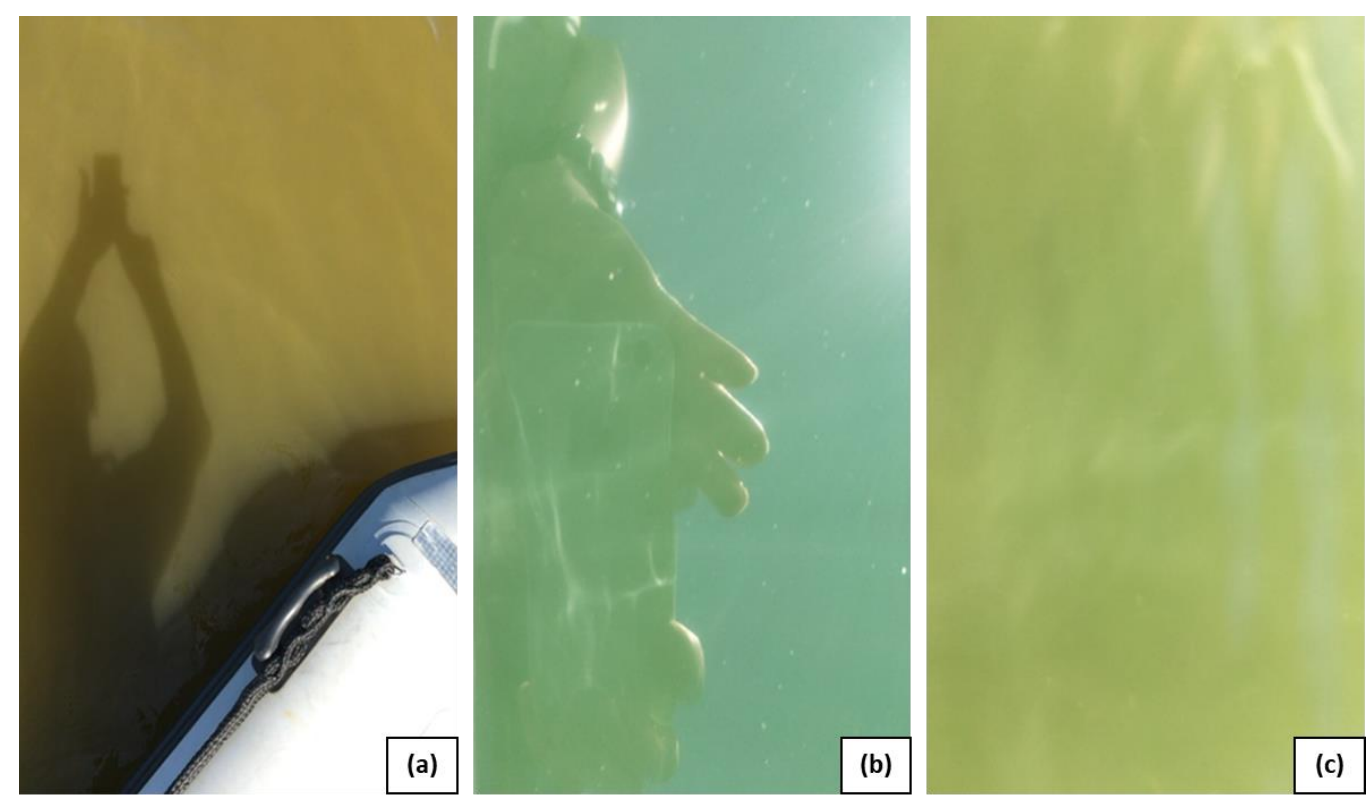

Figure 7. Three examples of images collected with the EoW App: Lake Atkinson (a), Lake St Clair station 6 (b) and Wivenhoe Dam station 1 (c).

A wide range of water colors were measured within this study, with the FUI ranging from 7 to 18. An initial inspection indicates that there is a higher occurrence of green waters within the sample of lakes chosen for this study, with values ranging mostly from 10 to 16. Lakes Atkinson and Dyer had higher FU_Values of 17 and 16, reflecting the green-yellow color of the waters. Lostock Reservoir and Ewen Maddock Dam both presented FU_Values of 17; these waters were darker in color and had higher CDOM concentrations present. The Brisbane River had a FU_Value of 18 and was significantly browner with higher SPM levels in comparison to the other sites. The site with the lowest FU_Value of 8 was Lake St Clair-this water body was clearer and bluer than the other sites measured and had lower CHL and SPM levels.

The processing of 36, instead of 16, subsections had limited impact on the 'best' hue angle and conversion to FU_Processed. In 23 out of 116 images, the FU scale shifted by plus or minus 1 unit; the overall correlation between the two values had a slope of 1.01, confirming that this is not a systematic effect, but merely a random effect whereby a slightly smaller or larger hue angle is found that just tips it into another FUI. In Figure 8, the FUI of WACODI (36 subsection, best value under sunny illumination conditions) is compared to the FUI entered by the observer. Two interesting aspects can be deduced from this histogram: first, a trained observer is indeed capable of accurately judging the FUI of the water from the smartphone screen; $78 \%$ of the results gave an FUI within one unit from the image FUI and only 3 out of 116 were 3 units off. Second, the distribution is somewhat skewed such that the image analysis overall provides a slightly smaller FUI than the observer. 


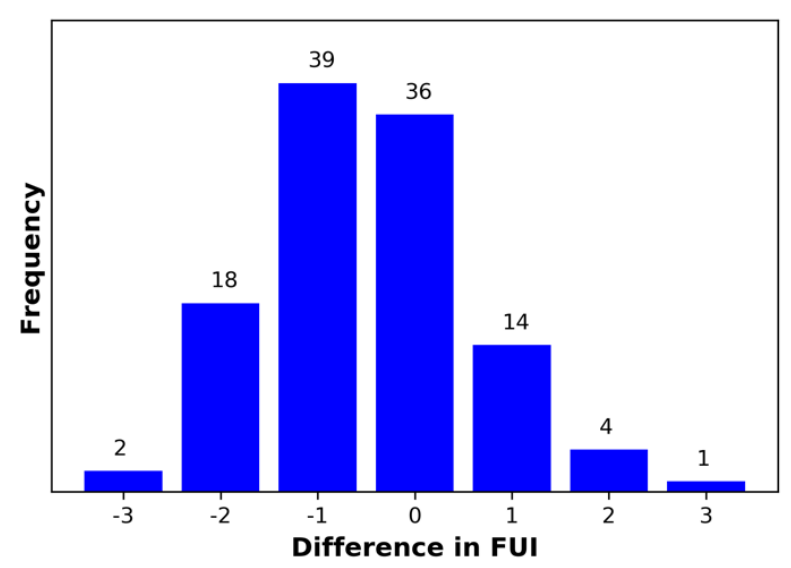

Figure 8. Frequency distribution of the difference in Forel-Ule index (FUI) between the WACODI-processed value $(\mathrm{P})$ and the FUI entered by the observer $(\mathrm{O})$.

The precision of EoW image information is analysed as follows: all images collected at each station were grouped together and the average hue angle was derived. Subsequently, the deviation from the average was calculated in degrees (the unit of hue angles). These results are shown in Figure 9. For a trained observer, it turns out that the collection and processing of an image is a process that provides stable results within a standard deviation of 3.15 degrees. To test the accuracy of WACODI, the image-derived hue angle was compared to the Satlantic hue angle. Note that per station, only one average Satlantic hue angle (Table S1) is available, while three to six EoW images were taken. When we assume that each EoW observation is an independent observation of a stable (over a period of approximately $30 \mathrm{~min}$ ) natural water that is characterized by the Satlantic hue angle, we find the correlation shown in Figure 10. A simple linear regression shows the slope close to 1.00 and an $R^{2}$ of 0.84 . This result is similar to the results reported for Dutch inland waters, based on WACODI-processing of images and Rrs spectra collected by a set of Ramses spectrometers [31]. Interestingly, this result did not improve or worsen if just the hue angles of centre subsections were correlated to the Satlantic values or if the overcast conditions were taken as standard illumination. This is demonstrated in Figure 11 which shows that overall the central hue angle is only slightly higher $(\mathrm{RC}=1.06)$ than the best hue angle. The outliers in this graph are all related to the complexity of the image collected, providing problems with the image processing. In fact, in the new WACODI-processing on the EoW server, the deviation of absolute difference best/centre hue angle should be less than 10 degrees in order to achieve the highest quality flag.

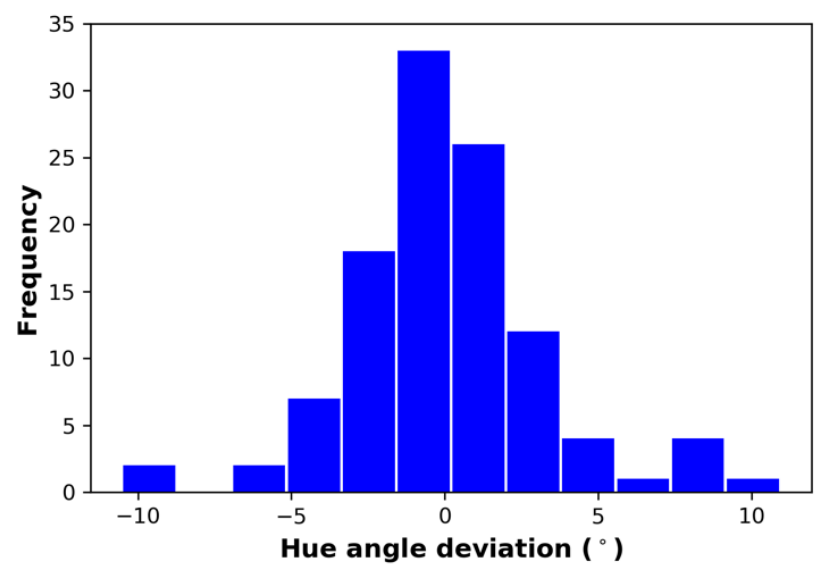

Figure 9. Frequency distribution of the difference in hue angle (degrees) between individual measurements and the mean per station. 


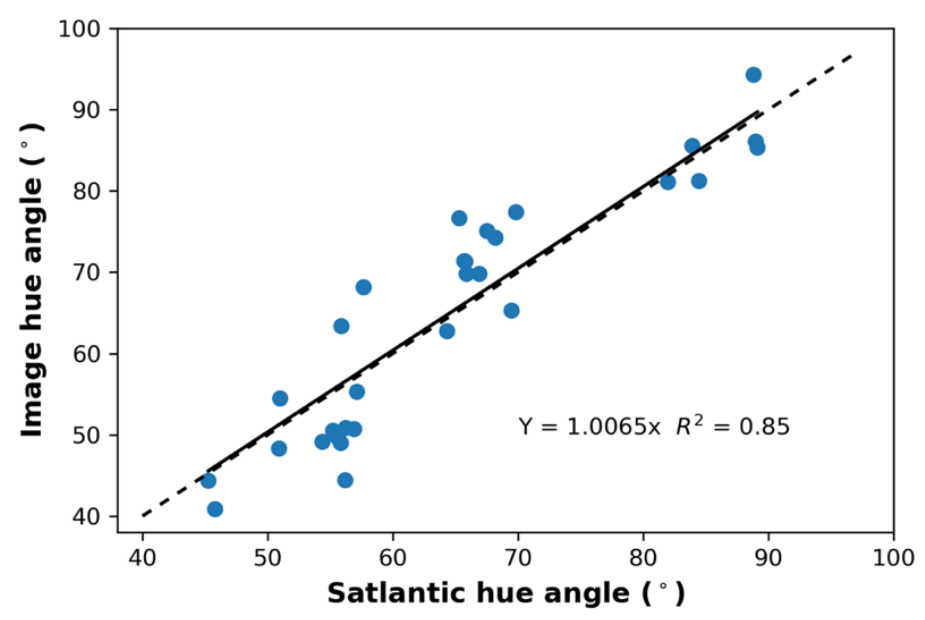

Figure 10. Correlation between hyperspectral (Satlantic) and mean EoW image (WACODI) hue angle. The solid line represents the regression through the data, and the dashed line represents the 1:1 relationship.

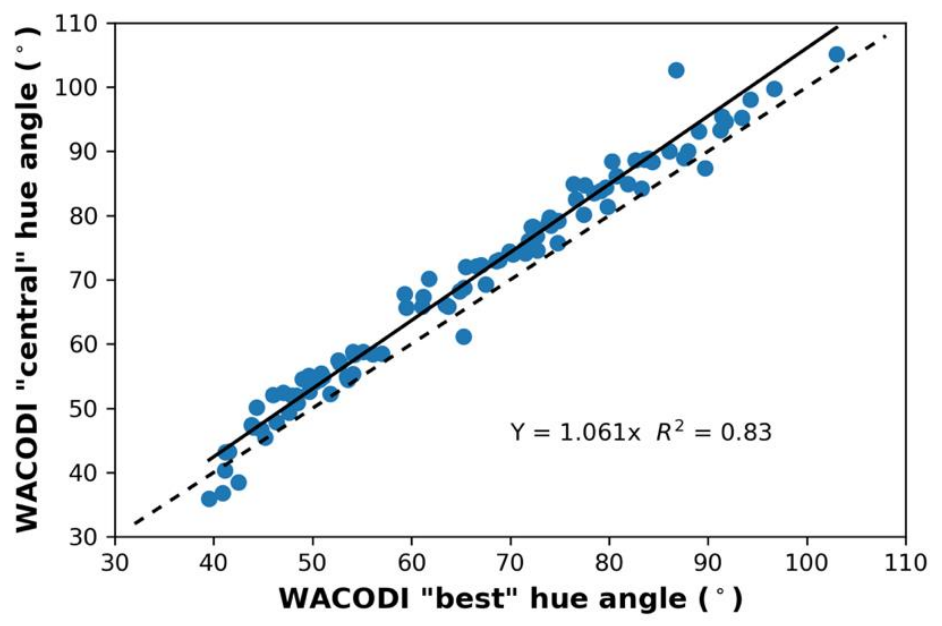

Figure 11. Relation between the hue angle derived from the center and best part of the image. The solid line represents the regression through the data, the dashed line represents the 1:1 relationship.

From this large set of data, collected from inland waters covering a wide range in composition, there is some encouragement for the accuracy and precision of the observed FUI and the processed FUI results. The average standard deviation for the replicates of the user selected FU_Value is 0.34 and 0.42 for the FU_Processed value. The slightly higher variation in the processed FUI values may be because the user may be more inclined to select the same color as previously selected, unless the images are significantly different. The results suggest that there is no need to take multiple measurements at each of the sites of interest as commonly the first image will be representative of the water color unless there is an issue with how it is taken. Our field experience suggests that the application is best used under clear and still water conditions to avoid the impacts of ripple reflection and precision issues do arise with very dark waters, windy conditions and fast-changing illumination conditions when it is partly cloudy.

\subsection{HC Performance}

During the field campaign, a total of 103 complete datasets were collected with the HC App. HC captures images, taken of the grey card, sky and water surface and the central $200 \times 200$ pixels are processed to derive Rrs (RGB). All relevant information, including location, time, solar zenith angle, viewing angles, integration times and intensity of the three images, plus derived Rrs and SPM 
concentration were downloaded and recorded. For this study, at least three replicates were taken at each site to determine the variability about a single measurement, although the HC app does not specify that three measurements should be taken at each site to reduce error. Figure 12 shows the variation between the three replicates taken, based on all the derived Rrs (RGB) values. It is evident that large errors did occur between the three replicates made in some water bodies and that the anomalies, when they occurred, are generally simultaneously present in each of the three bands. For Lostock01 replicate B, Somerset replicates $A$ and $C$ and Borumba replicates A and C zero was returned as a result.

Explanations for the causes of these variations include both how the photos were taken and the conditions they were taken under. Measurements taken at sites Lake Liddell 03, 05 and 06, Somerset, Borumba and Baroon Pocket Dam were made under patchy cloudy conditions. Despite the measurements being made in relatively quick succession, the variation between the replicates may be a result of moving clouds impacting on the red, blue and green channels. Clouds impact on the reflectance of the water as well as the intensity measured in the sky photo. Measurements taken under clear sunny conditions such as Lake Atkinson and Lake St Clair resulted in replicates with very low variation between measurements.

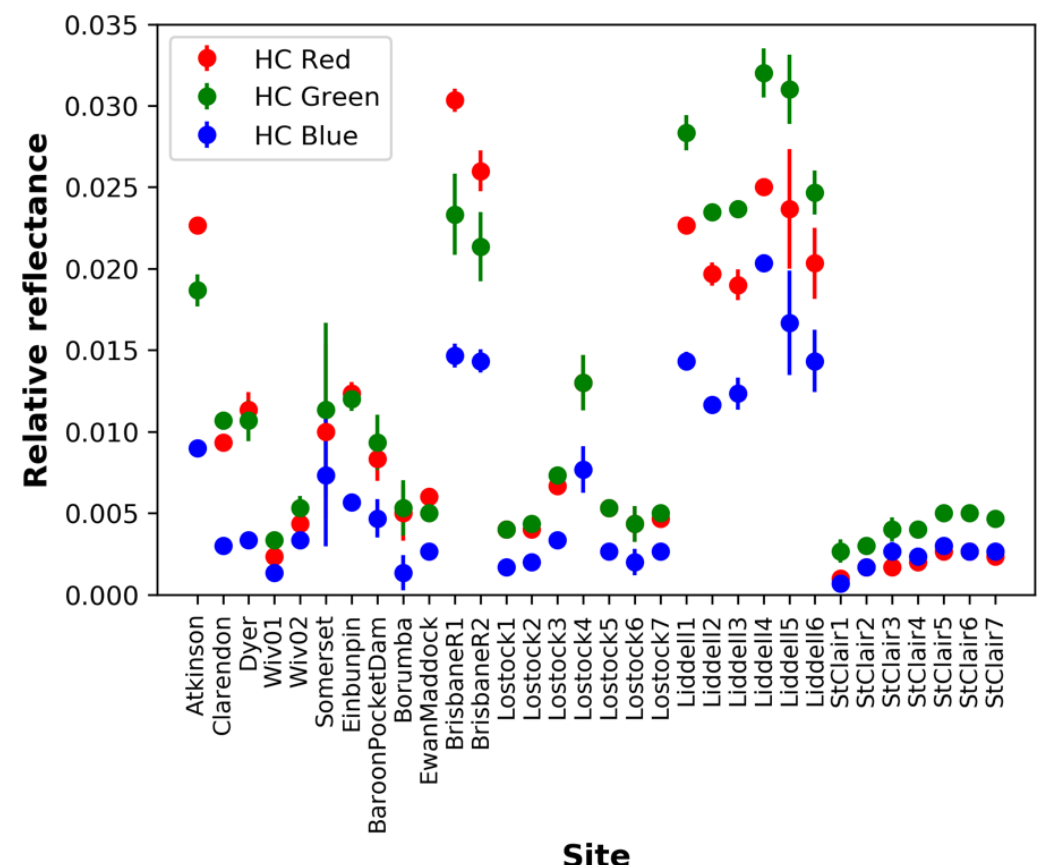

Figure 12. Summary of HC-derived Rrs (RGB) for all measurements, with variation between the three replicate HC RGB values expressed as bars indicating standard error.

The accuracy of the HC information was tested in the RGB bands. In Table S1, the Satlantic red band Rrs is given for 30 stations. Also given is the mean HC red band Rrs that is simply the mean value at each station, derived from 3 to 6 datasets. Figure 12 presents the Rrs (RGB) results derived from the images taken within HC. At first sight, the variation in peaks represents the wide range of water qualities measured within this study; indeed, the locations with higher peaks such as Lake Atkinson, Einbunpin Lagoon, Brisbane River, Lostock Dam and Lake Liddell represent sites which have higher turbidity or chlorophyll levels. However, Figure 13 demonstrates that the high values might have a different origin, because we find wide discrepancies when compared to the hyperspectral measurements. The results do not indicate any significant form of co-variation for each channel between the two sets of measurements for all bands. This indicates that the two measurement methods were not measuring the same quantity or that random errors are large with a high impact on the final mean values. Potential errors and remedies are discussed below. 


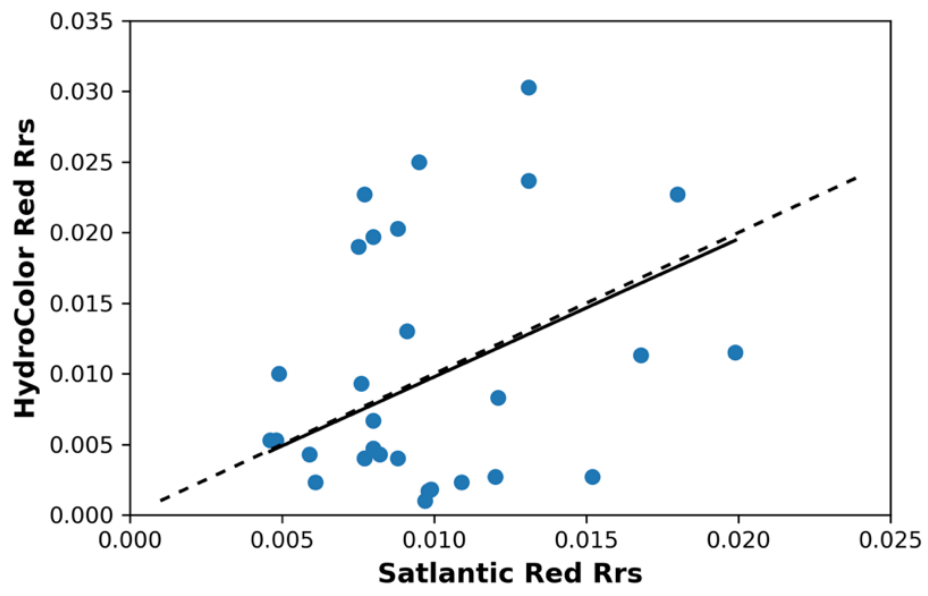

Figure 13. Relationship between the Satlantic and HC-derived Rrs in the Red channel. The solid line represents the regression through the data, and the dashed line represents the 1:1 relationship.

\subsection{HC and EoW Information on Water Composition}

The HC App calculates both turbidity (NTU) and concentration of suspended particular matter (SPM). In Figure 14, the relation is shown between the gravimetric measured and HC optically retrieved SPM concentrations; again, the $\mathrm{HC}$ result is the mean value of the 3-6 replicates at 30 stations. HC perform well in some water bodies at low SPM concentrations, but not others (e.g., Lake Liddell). Because SPM is retrieved from the red channel Rrs, the error in the red Rrs is obviously translated to errors in the retrieved SPM concentration. In answer to the question we postulated at the outset of this study ("how accurate is the HC conversion of Rrs to SPM for the large variation in Australian lakes, rivers and dams?"), based on these results, no firm answer can be given. No further correlations could be established between the HC results and concentrations of CHL or CDOM.

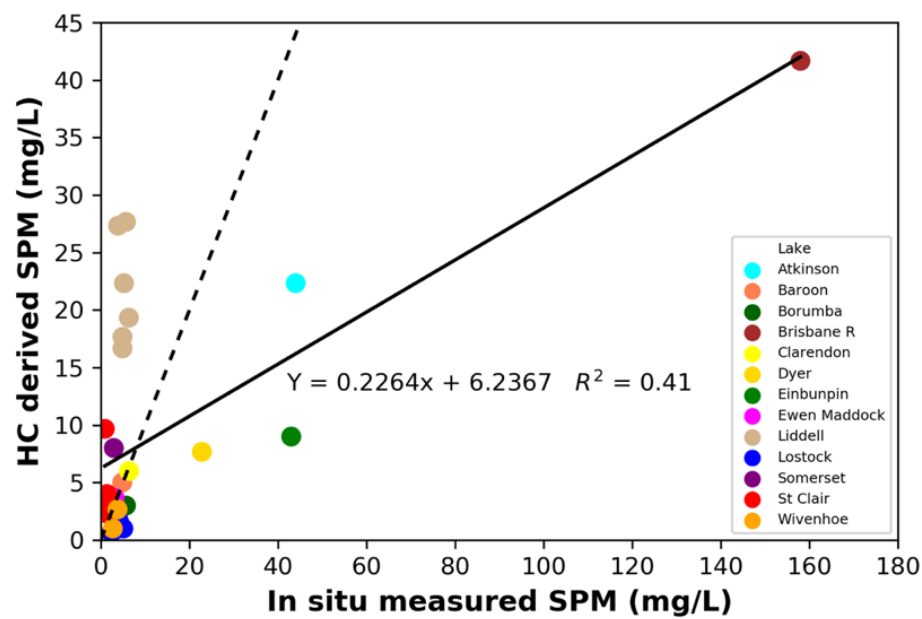

Figure 14. Relationship between in situ-measured suspended particulate matter (SPM) and HydroColor-derived SPM (mg/L) shown for individual lakes. The solid line represents the regression through the data, and the dashed line represents the 1:1 relationship.

The EoW app does not provide an estimate for water quality parameters but it is through the FU scale that the relative health of the water can be estimated. The scale range of 21 colors from indigo blue to brown, through blue-green, green and yellow colors, is indicative of the level of nutrients or algae present (1-5 FU), dissolved matter and sediment (6-9 FU), phytoplankton levels (10-13 FU), sediment (14-17 FU) and humic acids (18-21 FU). First, the observed FU_Value over the full range of values (8-18 FU) was simply correlated to in situ measurements of SDD, SPM and CHL. No significant 
relationships could be established. Second, we converted each FU_Value to the average SDD and CHL value derived at a global scale [57] (Tables A3 and A4), and then correlated to the in situ measurements. Again, no relation could be established. We conclude that no generic relation can be established between color and concentrations for these 13 very different Australian inland waters due to the absence of any relation between the three optically active substances.

\subsection{Spectral Integrity}

The two applications are difficult to compare, as HC uses a more robust approach to measure reflectance by taking three separate measurements of the grey card, sky and water surface, respectively, compared to the one single horizontal surface image taken by EoW. Despite this, the common ground between the two Apps was tested in a simple exercise. The average hue angle of the HC measurements is given in Table S1 and the procedure to convert the Rrs (RGB) of HC to x,y and subsequently to hue angle is described in Section 2.3. Because Figure 12 indicated that errors seem to be correlated for RGB, it might be that the shape of the reflection spectrum is less prone to errors than the absolute Rrs values. In Figure 15, the HC hue angle is related to the Satlantic hue angle. Although, the $\mathrm{R}^{2}$ is of the same order as the EoW results (Figure 10), the resulting trend line is very steep: the blue waters (e.g., Lake St. Clair) are extremely blue and the yellow-brown waters have an extreme red contribution, resulting in very low hue angles. The reason for this warrants further investigation. From this result, we conclude that $\mathrm{HC}$ has the potential to cover the full range of hue angles, but that first a correction must be applied to avoid overshooting of the spectral shape.

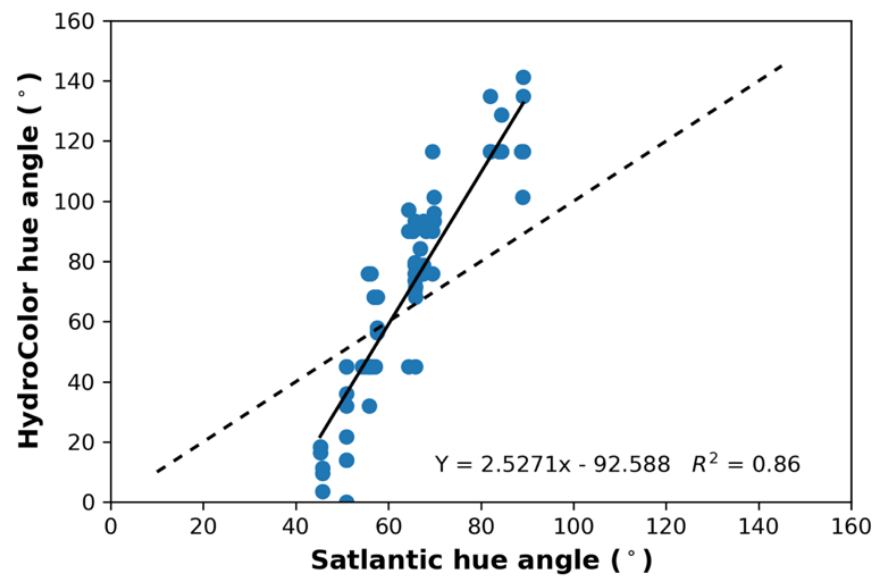

Figure 15. The hue angle of HC observations, plotted as a function of the Satlantic hue angle. The 1:1 reference line is shown, together with the steep linear trend line that fits the data. The solid line represents the regression through the data, and the dashed line represents the 1:1 relationship.

\section{Discussion}

The quality of Australian inland waters is under pressure and a dedicated monitoring effort is needed to evaluate changes and detect rapid changes. Algal blooms of blue-green algae, harmful to humans, animals and the local ecosystem, occur frequently in the lakes close to major cities of New South Wales and Queensland. Given the scale of the Australian continent, frequent in situ sampling of all major inland waters is almost an impossible task. Therefore, satellite observations, supported by a suite of in situ underwater and above-water automated sensors, are a good and welcome addition $[6,58]$. Optical close-range instruments can be applied to derive water quality parameters for monitoring purposes and for the validation of optical satellite data. Management authorities may implement the use of such sensors over traditional point measurements with the advantage being that estimates of water quality parameters are available almost instantly with high accuracy, enabling fast management decisions $[15,59]$. 
Citizen science offers a low-cost and potential solution for mapping environmental parameters such as water quality at a continental scale [21]. Although volunteered information has enhanced geographical and environmental data at virtually no cost, it has also prompted concerns in regards to the quality, reliability and the overall value of such data [60]. For this reason, we have tested the performance of the HC and EoW app and analysed the accuracy and precision of the collected information. Spectral measurements were taken with the Satlantic spectroradiometer as a reference surface reflectance measurement for the evaluation of the low-cost systems. A total of 13 inland waters with extreme differences in composition were visited and observations were carried out under strict professional protocols. A summary of the differences between the two apps can be found in [34].

The EoW App collects information on the water color, expressed in two related units; the hue angle and the FUI [31]. The observer reports a FUI, supported by a visual tool in the App. In the server and reported on the website, the FUI is also derived from the image taken by the observer. Overall, the results presented here suggest that the EoW app may measure the color of the water with good accuracy. In only $3 \%$ of the images, the observer and image reported a difference of more than 2 FUI units. The user-assigned values were $\sim 0.5$ FUI units higher than the processed values. Although this bias must be studied in more detail to find out its origin, we suggest five possible causes: (1) the difficulty of estimating the color of the digital image taken within the field. Although a shade cloth was used to view the phone screen in bright conditions, glare and reflectance from the water surface significantly impacted upon the ability to select a color which matched the image taken. (2) Saturation and brightness also impact upon how the color of water is perceived. One option is to take, one way or another, these effects into the color coding e.g., [29]. (3) Larger discrepancies occur at sites with patchy sky conditions. (4) It was difficult to obtain a matching FU_Value for darker waters such as Ewen Maddock Dam and Lostock Reservoir due to the relatively high contribution of reflection from the water surface. (5) The server software (WACODI) selects a section of the image that is most stable and has the lowest hue angle. Results presented in Figure 11 provide evidence that the hue angle, and thus the corresponding FUI in the image centre, where the observer has focussed, has a slightly higher value.

The color and the FUI should be considered as significant additional attributes of natural waters. Optically active constituents of the water, such as CHL, SPM and CDOM, together determine the overall reflection and its color. In this paper, we have shown that for Australian inland waters, there does not exist a simple inverse relation between color and CHL or SPM or Secchi disk depth. In a study by Wernand et al. [61], it was shown that in special cases, such as for the open ocean, a simple relation can be found for the FUI and CHL. Therefore, they were able to convert trends in the FUI over decades to trends in algal concentration. For inland waters we are at present aware of two publications that connect color to the state of water quality. At a very local scale of the Kesses Dam in Kenya, Ouma et al. [34] developed a local algorithm where small changes in SPM or CHL were correlated to changes in color, based on a local calibration. At the global scale, Wang et al. [28] demonstrated how the FUI derived from MODIS $500 \mathrm{~m}$ resolution images can be used to estimate the trophic state of lakes worldwide. This study is a milestone in color assessment of inland waters and can help to have a better understanding of the color derived from EoW.

The HC App has been developed as an 'easy to use interface' to calculate the water surface reflectance, using the three RGB channels of the phone camera as radiometers and following a measurement protocol that is standard for above-water hyperspectral measurement of water Rrs [32]. Despite the fact that HC is able to cover the three important components of the light field (total irradiation, sky radiation and water-leaving radiance), instead of only one in EoW, the HC Rrs compared poorly to the Satlantic spectroradiometer. The qualitative comparisons of RGB spectra from Satlantic, EoW and HC show, in many cases, large similarities but the presence of systematic and random errors in the retrieved information makes HC difficult to use as a monitoring device for SPM and turbidity. 
Because the whole concept of $\mathrm{HC}$ is physically sound, it certainly has the potential to become a key smartphone app for water managers. However, we suggest some further investigation, based on our field campaign and the results presented in [32-34]. Variation in the smartphone-derived Rrs (RGB) values may be expected due to the viewing geometry, detectors and spectral band-pass function used to record the amount of electromagnetic radiation and smartphone settings and processing methods of images.

The replicate and cloud cover assessment showed that the HC App performed poorer under patchy sky conditions resulting in a higher standard deviation between the replicates taken. This is inherent to the observation sequence that takes some time between the images, during which illumination conditions might have changed. This suggests that it is important to take multiple measurements, especially under cloudy conditions to detect and filter out the major outliers. Until recently, information on smartphone spectral response functions was lacking and we were dependant on the manufacturer to make a proper conversion of the raw counts in RGB to the sRGB format. Thereby, errors can be introduced that depend on illumination and reflection properties. Recently, Burggraaff et al. [62] presented a detailed laboratory study of the characteristics of smartphone cameras, including the spectral response function. As a final remark, this study and the previous study of the EoW results [31] found no major deviations in the sRGB products provided by Android or iPhone cameras. The iPhone camera setting during our campaign sometimes resulted in extremely low or zero values in the blue band. This has also been detected by Yang et al. [33], who also could not find an explanation for this behaviour.

The most important error in the overall post processing steps in the HC App seems to be the handling of the non-linearity of the image material. [32] tested the linearity of the iPhone in the lab and concluded that the impact of sRGB conversion and Gamma correction is small. However, there are a number of other findings which contradict this: [62] demonstrated that the Gamma correction is significant, varying between 2.2 and 2.45 with the color (RGB) and position in the image. Furthermore, in WACODI, a Gamma index of 2.2 is adopted for all positions and all bands; this correction is essential to retrieve reliable hue angles. The results of this campaign also demonstrate that HC Rrs derived from waters with a strong blue (hue angle larger than 90 degrees) or red (hue angle less than 40 degrees) color are exaggerated. This is exactly the expected impact of the Gamma expansion in the processing from raw image to sRGB. Finally, it was also found that for the clear blue waters between Vancouver and Vancouver Island in Canada, the dynamic range in blue/green ratios of the Ramses spectrometers was systematically different from the HC Rrs blue/green ratio [33].

\section{Conclusions}

This analysis has been undertaken to test the usability, strengths and weaknesses of the EyeOnWater and HydroColor Apps as citizen science methods to obtain and portray information relevant for water quality monitoring.

After extensive testing on multiple water bodies, there is a degree of confidence that the modern FU scale presented within the EoW App is appropriate and provides a fairly accurate estimation of the condition of the water. The testing has shown that an observer can capture images with the App, and select the corresponding color of the water body to provide an accurate estimation of the quality of the water. This analysis has shown that, although highly useful in its own right, the relationship between water color and water quality is complex and that the EoW App should not be used as a surrogate for other water quality variables of interest; this is not the intention of the FU scale. The FU scale may simply be an indication of the visual appearance of the water surface to monitor over the seasons and prompt further laboratory water quality testing.

The results for the HC App were less conclusive than expected. In these difficult circumstances, with sun almost near zenith and a wide range of waters, from highly reflective (Brisbane River) to highly absorptive (Lake St Clair), the results were prone to errors. Before an assessment can be made of 
the applicability of the SPM algorithm in the App, it is essential to first reduce the errors in the retrieved Rrs. It remains important to take multiple measurements, especially under cloudy conditions.

Finally, it is important to note that both the HydroColor and EyeOnWater Apps were only tested by two users. In order to gauge an understanding of the influence of the user on the quality of the results, these Apps should be tested on a larger group of users.

Supplementary Materials: The following are available online at http://www.mdpi.com/2072-4292/12/10/1578/s1, Figure S1: Mean raw spectra for panel and water surface measurements made at Station 6 on Lake Liddell, 02.03.2016, presented together with standard deviation and coefficient of variation, Figure S2: Model outcomes to show changes in hue angle when a spectrometer is lowered to $5 \mathrm{~cm}$ depth in natural waters, Table S1: Summary of the basic measurements from all stations.

Author Contributions: The general set up of the measurement campaign was developed by T.J.M. R.O. and T.J.M. carried out the field measurements and H.J.v.d.W. assisted with data handling with the support of other CSIRO staff. This work formed RO's extended Honours study under the supervision of T.J.M. and Stuart Phinn. R.O., T.J.M. and H.J.v.d.W. all interpreted the dataset and wrote the article. All authors have read and agreed to the published version of the manuscript.

Funding: This project was in part supported by funding from the New South Wales Office of the Premier in a project entitled "Early detection of algal blooms using remote sensing". HvdW received travel support from the "Citizen Science helping improve satellite detection of water quality" project supported by the Australian Federal Department of Industry, Science, Energy and Resources (grant number CSG56397), led by Janet Anstee (CSIRO).

Acknowledgments: We wish to thank Heidi Franklin, Janet Anstee and Hannelie Botha (all from CSIRO) for their assistance in the field work and laboratory training. Access to South Eastern Queensland water reservoirs was kindly granted by Seqwater (seqwater.com.au); our thanks to Deb Gale and Cameron Veal for useful discussions. We also acknowledge the support of Adrian D'Alessandro (CSIRO) in the conversion of WACODI to Python script.

Conflicts of Interest: The authors declare no conflict of interest.

\section{References}

1. Khalil, B.; Ouarda, T.B.M.J.; St-Hilaire, A.; Chebana, F. A statistical approach for the rationalization of water quality indicators in surface water quality monitoring networks. 2010. J. Hydrol. 2010, 386, 173-185. [CrossRef]

2. Paerl, H.W.; Huisman, J. Climate change: A catalyst for global expansion of harmful cyanobacterial blooms. Environ. Microbiol. Rep. 2009, 1, 27-37. [CrossRef]

3. Fritz, S.; See, L.; Carlson, T.; Hakley, M.; Oliver, J.L.; Fraisl, D.; Mondardini, R.; Brocklehurst, M.; Shanley, L.A.; Schade, S.; et al. Citizen Science and the United Nations Sustainable Development Goals. Nat. Sustain. 2019, 2, 922-930. [CrossRef]

4. Dekker, A.G. Detection of Optical Water Quality Parameters for Eutrophic Waters by High Resolution Remote Sensing. Ph.D. Thesis, Vrije University, Amsterdam, The Netherland, 1993.

5. Olmanson, L.G.; Brezonik, P.L.; Bauer, M.E. Airborne hyperspectral remote sensing to assess spatial distribution of water quality characteristics in large rivers: The Mississippi River and its tributaries in Minnesota. Remote Sens. Environ. 2013, 130, 254-265. [CrossRef]

6. Hestir, E.L.; Brando, V.E.; Bresciani, M.; Giardino, C.; Matta, E.; Villa, P.; Dekker, A.G. Measuring freshwater aquatic ecosystems: The need for a hyperspectral global mapping satellite mission. Remote Sens. Environ. 2015, 167, 181-195. [CrossRef]

7. Busch, J.A.; Zielinski, O.; Cembella, A.D. Subsea Optics and Imaging. In Subsea Optics and Imaging; Elsevier: Amsterdam, The Netherlands, 2013. [CrossRef]

8. Gons, H.J.; Rijkeboer, M.; Bagheri, S.; Ruddick, K.G. Optical teledetection of chlorophyll a in estuarine and coastal waters. Environ. Sci. Technol. 2000, 34, 5189-5192. [CrossRef]

9. Gitelson, A.; Gurlin, D.; Moses, W.J.; Barrow, T. A bio-optical algorithm for the remote estimation of the chlorophyll- a concentration in case 2 waters. Environ. Res. Lett. 2009, 4, 45003. [CrossRef]

10. Matthews, M.W. A current review of empirical procedures of remote sensing in inland and near-coastal transitional waters. Int. J. Remote Sens. 2011, 32, 6855-6899. [CrossRef]

11. Dekker, A.G.; Vos, R.J.; Peters, S.W.M. Analytical algorithms for lake water TSM estimation for retrospective analyses of TM and SPOT sensor data. Int. J. Remote Sens. 2002, 23, 15-35. [CrossRef] 
12. Nechad, B.; Ruddick, K.G.; Park, Y. Calibration and validation of a generic multisensor algorithm for mapping of total suspended matter in turbid waters. Remote Sens. Environ. 2010, 114, 854-866. [CrossRef]

13. Al-Kharusi, E.S.; Tenenbaum, D.E.; Abdi, A.M.; Kutser, T.; Karlsson, J.; Bergström, A.-K.; Berggren, M. Large-Scale Retrieval of Coloured Dissolved Organic Matter in Northern Lakes Using Sentinel-2 Data. Remote Sens. 2020, 12, 157. [CrossRef]

14. Dekker, A.; Hestir, E. Evaluating the Feasibility of Systematic Inland Water Quality Monitoring with Satellite Remote Sensing; CSIRO: Canberra, Australia, 2012.

15. Hommersom, A.; Kratzer, S.; Laanen, M.; Ansko, I.; Ligi, M.; Bresciani, M.; Giardino, C.; Beltrán-Abaunza, J.M.; Moore, G.; Wernand, M.; et al. Intercomparison in the field between the new WISP-3 and other radiometers (TriOS Ramses, ASD FieldSpec, and TACCS). J. Appl. Remote Sens. 2012, 6, 63615. [CrossRef]

16. Hunter, P.D.; Tyler, A.N.; Carvalho, L.; Codd, G.A.; Maberly, S.C. Hyperspectral remote sensing of cyanobacterial pigments as indicators for cell populations and toxins in eutrophic lakes. Remote Sens. Environ. 2010, 114, 2705-2718. [CrossRef]

17. Li, L.; Sengpiel, R.E.; Pascual, D.L.; Tedesco, L.P.; Wilson, J.S.; Soyeux, E. Using hyperspectral remote sensing to estimate chlorophyll- a and phycocyanin in a mesotrophic reservoir. Int. J. Remote Sens. 2010, 31, 4147-4162. [CrossRef]

18. Le, C.; Li, Y.; Zha, Y.; Sun, D.; Huang, C.; Zhang, H. Remote estimation of chlorophyll a in optically complex waters based on optical classification. Remote Sens. Environ. 2011, 115, 725-737. [CrossRef]

19. O'Donnell, D.M.; Effler, S.W.; Strait, C.M.; Peng, F.; Perkins, M. Remote sensing reflectance in the Great Lakes: In situ measurements, closure analyses, and a forward model. J. Great Lakes Res. 2013, 39, 137-150. [CrossRef]

20. Kudela, R.M.; Palacios, S.L.; Austerberry, D.C.; Accorsi, E.K.; Guild, L.S.; Torres-Perez, J. Application of hyperspectral remote sensing to cyanobacterial blooms in inland waters. Remote Sens. Environ. 2015, 167, 196-205. [CrossRef]

21. Busch, J.A.; Badají, R.; Ceccaroni, L.; Friedrichs, A.; Piera, J.; Simon, C.; Thijsse, P.; Wernand, M.; Van der Woerd, H.J.; Zielinski, O. Citizen bio-optical observations from coast- and ocean and their compatibility with ocean colour satellite measurements. Remote Sens. 2016, 8, 879. [CrossRef]

22. Brando, V.E.; Lovell, J.L.; King, E.A.; Boadle, D.; Scott, R.; Schroeder, T. The Potential of Autonomous Ship-Borne Hyperspectral Radiometers for the Validation of Ocean Color Radiometry Data. Remote Sens. 2016, 150. [CrossRef]

23. Lymburner, L.; Botha, B.; Hestir, E.; Anstee, J.; Sagar, S.; Dekker, A.; Malthus, T. Landsat 8: Providing continuity and increased precision for measuring multi-decadal time series of total suspended matter. Remote Sens. Environ. 2016, 185, 108-118. [CrossRef]

24. Malthus, T.J.; Lehmann, E.; Ho, X.; Botha, E.; Anstee, J. Implementation of a satellite based inland water algal bloom alerting system using analysis ready data. Remote Sens. 2019, 11, 2954. [CrossRef]

25. Wernand, M.R.; Hommersom, A.; Van der Woerd, H.J. MERIS-based ocean colour classification with the discrete Forel-Ule scale. Ocean Sci. 2013, 9, 477-487. [CrossRef]

26. Van der Woerd, H.J.; Wernand, M.R. True colour classification of natural waters with medium-spectral resolution satellites: SeaWiFS, MODIS, MERIS and OLCI. Sensors 2015, 15, 25663-25680. [CrossRef] [PubMed]

27. Van der Woerd, H.J.; Wernand, M.R. Hue angle product for low to medium spectral resolution optical satellite sensors. Remote Sens. 2018, 10, 180. [CrossRef]

28. Wang, S.; Junsheng, L.; Zhang, B.; Spyrakos, E.; Tyler, A.N.; Shen, Q.; Zhang, F.; Kutser, T.; Lehmann, M.K.; $\mathrm{Wu}, \mathrm{Y}$; et al. Trophic state assessment of global inland waters using a MODIS-derived Forel-Ule index. Remote Sens. Environ. 2018, 217, 444-460. [CrossRef]

29. Lehmann, M.K.; Nguyen, U.; Allan, M.; Van der Woerd, H.J. Colour classification of 1486 lakes across a wide range of optical water types. Remote Sens. 2018, 10, 1273. [CrossRef]

30. Giardino, C.; Koks, K.; Bolpagni, R.; Luciani, G.; Candiani, G.; Lehmann, M.K.; Van der Woerd, H.J.; Bresciani, M. The color of water from space: A case study for Italian lakes from Sentinel-2. In Earth Observation and Geospatial Analysis; Intech Open: London, UK, 2019. [CrossRef]

31. Novoa, S.; Wernand, M.R.; Van der Woerd, H.J. WACODI: A generic algorithm to derive the intrinsic color of natural waters from digital images. Limnol. Oceanogr. Methods 2015, 13, 697-711. [CrossRef]

32. Leeuw, T.; Boss, E. The HydroColor App: Above water measurements of remote sensing reflectance and turbidity using a smartphone camera. Sensors 2018, 18, 256. [CrossRef] 
33. Yang, Y.; Cowen, L.L.E.; Costa, M. Is ocean reflectance acquired by citizen scientists robust for science applications? Remote Sens. 2018, 10, 835. [CrossRef]

34. Ouma, Y.O.; Waga, J.; Okech, O.; Lavisa, O.; Mbuthia, D. Estimation of reservoir bio-optical water quality parameters using smartphone sensor apps and Landsat ETM+: Review and comparative experimental results. J. Sens. 2018, 3490757. [CrossRef]

35. Fargoin, G.S.; Mueller, J.L. Ocean Optics Protocols for Satellite Ocean Color Sensor Validation, 3rd ed.; NASA Goddard Space Flight Center: Greenbelt, MD, USA, 2002.

36. Tilstone, G.H.; Moore, G.F.; Doerffer, R.; Røttgers, R.; Ruddick, K.G.; Pasterkamp, R.; Jørgensen, P.V. Regional Validation of MERIS Chlorophyll products in North Sea REVAMP Protocols. In Proceedings of the Working Meeting on MERIS and AATSR Calibration and Geophysical Validation (ENVISAT MAVT-2003), Frascati, Rome, Italy, 20-24 October 2003; ESA Special Publication WPP-233: Noordwijk, The Netherlands, 2002; pp. 1-77.

37. Clementson, L.A.; Parslow, J.S.; Turnbull, A.R.; Mckenzie, D.C.; Rathbone, C.E. Optical properties of waters in the Australasian sector of the Southern Ocean. J. Geophys. Res. Oceans 2001, 106, 31611-31625. [CrossRef]

38. Zibordi, G.; Talone, M. On the equivalence of near-surface methods to determine the water-leaving radiance. Opt. Express 2020, 28, 3200. [CrossRef] [PubMed]

39. Ruddick, K.G.; Vos, K.; Boss, E.; Castagna, A.; Frouin, R.; Gilerson, A.; Hieronymi, M.; Johnson, B.C.; Kuusk, J.; Lee, Z.; et al. A Review of Protocols for Fiducial Reference Measurements of Water-Leaving Radiance for Validation of Satellite Remote-Sensing Data over Water. Remote Sens. 2019, 11, 2198. [CrossRef]

40. Simis, S.G.H.; Peters, S.W.M.; Gons, H.J. Remote sensing of the cyanobacterial pigment phycocyanin in turbid inland water. Limnol. Oceanogr. 2005, 50, 237-245. [CrossRef]

41. Ruiz-Verdu, A.; Simis, S.G.H.; De Hoyos, C.; Gons, H.J.; Peña-Martínez, R. An evaluation of algorithms for the remote sensing of cyanobacterial biomass. Remote Sens. Environ. 2008, 112, 3996-4008. [CrossRef]

42. Gitelson, A.A.; Dall'Olmo, G.; Moses, W.; Rundquist, D.C.; Barrow, T.; Fisher, T.R.; Gurlin, D.; Holz, J. A simple semi-analytical model for remote estimation of chlorophyll-a in turbid waters-Validation. Remote Sens. Environ. 2008, 112, 3582-3593. [CrossRef]

43. Li, L.; Li, L.; Song, K. Remote sensing of freshwater cyanobacteria: An extended IOP Inversion Model of Inland Waters (IIMIW) for partitioning absorption coefficient and estimating phycocyanin. Remote Sens. Environ. 2015, 157, 9-23. [CrossRef]

44. Liu, G.; Lin, L.; Song, K.; Li, Y.; Lyu, H.; Wen, Z.; Fang, C.; Bi, S.; Sun, X.; Wang, Z.; et al. An OLCI-based algorithm for semi-empirically partitioning absorption coefficient and estimating chlorophyll a concentration in various turbid case-2 waters. Remote Sens. Environ. 2020, 239, 111648. [CrossRef]

45. Ogashawara, I.; Mishra, D.R.; Mishra, S.; Curtarelli, M.P.; Stech, J.L. A Performance Review of Reflectance Based Algorithms for Predicting Phycocyanin Concentrations in Inland Waters. Remote Sens. 2013, 5, 4774-4798. [CrossRef]

46. Spyrakos, E.; O’Donnell, R.; Hunter, P.D.; Miller, C.; Scott, M.; Simis, S.G.H.; Niel, C.; Barbosa, C.; Binding, C.E.; Bradt, S.; et al. Optical types of inland and coastal waters. Limnol. Oceanogr. 2017, 63, 846-870. [CrossRef]

47. Kirk, J.T. Light and Photosynthesis in Aquatic Ecosystems, 3rd ed.; Cambridge University Press: Cambridge, NY, USA, 2011.

48. CIE. Commission Internationale de l'Éclairage Proceedings, 1931; Cambridge University Press: Cambridge, UK, 1932; pp. 19-29.

49. Novoa, S.; Wernand, M.R.; Van der Woerd, H.J. The Forel-Ule scale revisited spectrally: Preparation protocols, transmission measurements and chromaticity. J. Eur. Opt. Soc. RP 2013, 8, 13057. [CrossRef]

50. Leeuw, T. Crowdsourcing Water Quality Data Using the iPhone Camera; Paper 2118; University of Maine: Orono, ME, USA, 2014; Available online: http://digitalcommons.library.umaine.edu/etd/2118 (accessed on 24 January 2020).

51. Garaba, S.; Voss, D.; Wollschlager, J.; Zielinski, O. Modern approaches to shipborne ocean color remote sensing. Appl. Opt. 2015, 54, 3602-3612.

52. Mueller, J.L.; Fargion, G.S.; Mcclain, C.R.; Mueller, J.L.; Morel, A.; Frouin, R.; Davis, C.; Arnone, R.; Carder, K.; Steword, R.G.; et al. NASA/TM-2003-Ocean Optics Protocols for Satellite Ocean Color Sensor Validation, Revision 4, Volume III: Radiometric Measurements and Data Analysis Protocols; NASA: Washington DC, USA, 2003.

53. Mobley, C.D. Estimation of the remote-sensing reflectance from above-surface measurements. Appl. Opt. 1999, 38, 7442-7455. [CrossRef] [PubMed] 
54. Pascale, D. A review of RGB color spaces... from xyY to $\mathrm{R}^{\prime} \mathrm{G}^{\prime} \mathrm{B}^{\prime}$. 2003. Babel Color. pp. 1-35. Available online: www.babelcolor.com (accessed on 24 January 2020).

55. Lindell, L.T.; Pierson, D.; Premazzi, G.; Zillioli, E. Manual for Monitoring European Lakes Using Remote Sensing Techniques; Joint Research Centre European Commission: Ispra, Italy, 1999; ISBN 978-9282853900.

56. Van der Woerd, H.J.; Pasterkamp, R. Mapping of the North Sea turbid coastal waters using SeaWiFS data. Can. J. Remote Sens. 2004, 30, 44-53. [CrossRef]

57. Pitarch, J.; Van der Woerd, H.J.; Brewin, R.J.W.; Zielinsky, O. Optical properties of Forel-Ule water types deduced from 15 years of global satellite ocean color observations. Remote Sens. Environ. 2018, 231, 111249. [CrossRef]

58. Malthus, T.J.; Lehmann, E.; Ho, X.; Gensemer, S.; Anstee, J.; Botha, E.; Brayan, J.; Bowling, L.; Shaikh, M.; Ohmsen, R.; et al. An algal bloom early warning system for NSW using satellite and near-surface observations. In Proceedings of the OzWater17 Conference, 16-18 May 2017; Australian Water Association: Municipality, Australia. Available online: https:/awa.sharefile.com/share?\#/view/s69fcd4a5d344354b (accessed on 23 February 2020).

59. Bowling, L.; Shaikh, M.; Brayan, J.; Malthus, T. An evaluation of a handheld spectroradiometer for the near real-time measurement of cyanobacteria for bloom management purposes. Environ. Monit. Assess. 2017, 189, 495. [CrossRef]

60. Flanagin, A.J.; Metzger, M.J. The credibility of volunteered geographic information. GeoJournal 2008, 72, 137-148. [CrossRef]

61. Wernand, M.R.; Van der Woerd, H.J.; Gieskes, W.W.C. Trends in Ocean Colour and Chlorophyll Concentration from 1889 to 2000, Worldwide. PLoS ONE 2013, 8. [CrossRef]

62. Burggraaff, O.; Schmidt, N.; Zamorano, J.; Puly, K.; Pascual, S.; Tapia, C.; Spyrakos, E.; Snik, F. Standardized spectral and radiometric calibration of consumer cameras. Opt. Express. 2019, 19075. [CrossRef]

(C) 2020 by the authors. Licensee MDPI, Basel, Switzerland. This article is an open access article distributed under the terms and conditions of the Creative Commons Attribution (CC BY) license (http://creativecommons.org/licenses/by/4.0/). 